\title{
Modeling Microbunching from Shot Noise Using Vlasov Solvers
}

\author{
M. Venturini and A. Zholents \\ Lawrence Berkeley National Laboratory, Univ. of California \\ 1 Cyclotron Rd, Berkeley CA, 94720, USA
}

\begin{abstract}
Unlike macroparticle simulations, which are sensitive to unphysical statistical fluctuations when the number of macroparticles is smaller than the bunch population, direct methods for solving the Vlasov equation are free from sampling noise and are ideally suited for studying microbunching instabilities evolving from shot noise. We review a 2D (longitudinal dynamics) Vlasov solver we have recently developed to study the microbunching instability in the beam delivery systems for x-ray FELs and present an application to FERMI@Elettra. We discuss, in particular, the impact of the spreader design on microbunching.
\end{abstract}

Key words: FEL, microbunching instability, space charge PACS: 29.27.Bd, 41.60.Ap, 41.60.Cr, 52.65.Ff

\section{Introduction}

The microbunching instability [1/2/344 in the beam delivery systems for x-ray FELs can severely degrade the beam quality thus compromising performance. The physical processes causing the instability are very much the same as those being exploited in the undulator for lasing: particle self-fields cause energy changes in the beam that result into density fluctuations as the particles travels through bending elements. In turn, these density fluctuations seed larger energy variations further feeding the amplification process. Because the most fundamental source of fluctuations causing the instability is shot noise using macroparticles to simulate the dynamics of an electron bunch may be problematic unless the number of macroparticles used approaches the actual number of electrons [5]. Direct

Email address: mventurini@lbl.gov, aazholents@lbl.gov (M. Venturini and A. Zholents).
Vlasov solver methods offer an interesting alternative. By representing the beam density functions in phase space on grids these methods are immune to sampling noise and may allow for a more convincing characterization of genuinely physical instabilities.

We have recently developed a 2D solver [6] specifically devoted to the study of single pass system of relevance for x-ray FELs. This extends previous work [7] where Valsov solver techniques were used to characterize the CSR-induced microbunching instabilities in storage rings. Work by other investigators using direct methods is also ongoing [8].

The solver follows the evolution of the beam density in the longitudinal phase space on the assumption that changes in the transverse phase-space density due to collective effects are negligible or have negligible consequences on the longitudinal dynamics. The mixing effect of a finite transverse emittance in the longitudinal phase space, which is present when the beam travels through a dispersive region, is modelled in a heuristic way by insertion of a low-pass 


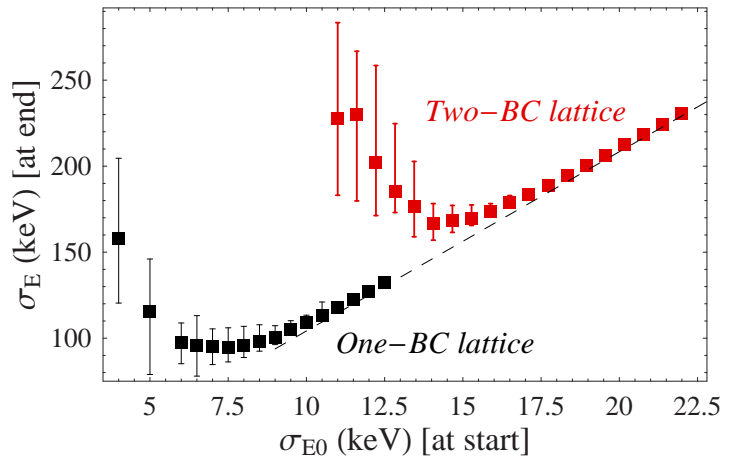

Fig. 1. Beam uncorrelated rms energy spread at the exit vs that at the entry of the linac for two FERMI lattice configurations. The boxes are averages value over several realizations of the initial beam perturbation modelling shot noise (the error bars span the full data range); $\varepsilon_{x}=\varepsilon_{y}=1 \mu \mathrm{m}$, $I_{f}=0.8 \mathrm{kA}$.

filter in the kernel for the evaluation of the collective force. The solver uses simplified models for determination of the collective effects relevant for the microbunching instability, (namely longitudinal space charge and coherent synchrotron radiation, CSR). In its present implementation it ignores other collective effects that may be otherwise important but are not relevant for the microbunching instability.

CSR is treated in the free-space model of a beam in uniform circular motion 26]. Longitudinal space charge is described by the impedance per unit length 936

$\hat{Z}(k)=\frac{i Z_{0}}{\pi \gamma r_{b}} \frac{1-x K_{1}(x)}{x}, \quad$ with $x=k r_{b} / \gamma ;$

where $\gamma$ is the relativistic factor, $K_{1}(x)$ the modified Bessel function and $Z_{0}=120 \pi \Omega$, the vacuum impedance. This formula applies to a bunch with transversally uniform density and circular cross section of radius $r_{b}$ in free space and yields the electric field on the beam axis. To model a transversally gaussian beam with rms sizes $\sigma_{x}$ and $\sigma_{y}$ we set $r_{b}=$ $1.7\left(\sigma_{x}+\sigma_{y}\right) / 2,310$.

Periodic boundary conditions are enforced in the longitudinal space coordinate. This is an effective way to reduce the size of the grid supporting the beam density function and hence the computation time without significantly affecting the accuracy of the modelling provided that the region of phase space selected for gridding is large compared to the length scale over which the instability develops. Shot noise is modelled by a suitable random perturbation of the beam density in phase space at the start of the simulation. For more details on the

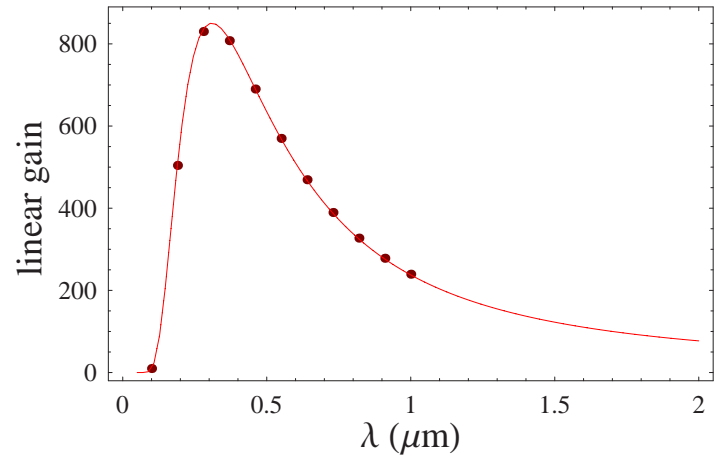

Fig. 2. The small-amplitude gain function for the microbunching instability from the end of the BC through the spreader in the One-BC lattice (un-optimized spreader design) shows a large gain in the sub $\mu \mathrm{m}$ region. The numerical results using the solver (dots) agree well with linear theory (solid line).

solver we refer to 64 .

\section{Application to FERMI}

The FERMI project [1] is in its final design stage. A few unresolved issues still remain regarding some aspects of the lattice for the beam delivery system. The design goal is for a machine delivering a beam with less than $150 \mathrm{keV}$ (uncorrelated) energy spread (with a normalized rms transverse emittance of $1.5 \mu \mathrm{m}$ ). Control of the microbunching instability is an important factor weighing in the final choice of the lattice. A 'laser heater' will be in place located right after the injector to increase the beam uncorrelated energy spread. The purpose of a larger beam energy spread early on is to boost phase-space mixing and therefore smoothen the microbunching as the beam travels through the bunch compressors. However, simulations show that the required 'heating' for the two bunch-compressor (BC) lattice, the current baseline, may be incompatible with the design goal for the energy spread. These simulations have prompted consideration of a lattice with a single bunch compressor providing at once the required compression factor $\simeq 10$ needed to achieve $I_{f}=$ $0.8 \mathrm{kA}$ peak current at extraction. In the One-BC lattice the bunches travel a longer section of the linac with higher peak current but also with larger energy spread. As it happens, the beneficial mixing from the latter prevails and the net effect is to noticeably reduce the instability. Evidence of the One-BC lattice better performance is shown in Fig. 1 where we plot the uncorrelated rms energy spread $\sigma_{E}$ at the end of the linac corresponding to various choices of 


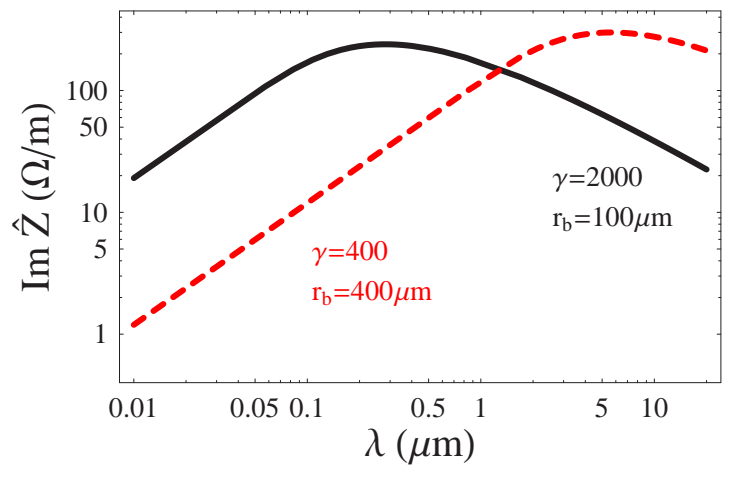

Fig. 3. Space charge impedance vs. wavelength $\lambda=2 \pi / k$ for choices of beam parameters corresponding to the early part (red dashed curve) and end of the linac (black line).

the rms energy spread $\sigma_{E 0}$ at the location of the laser heater. The beam is assumed to have an initially gaussian energy density. For a given $\sigma_{E 0}$ the resulting $\sigma_{E}$ depends on the seed for the random noise placed on the beam density at the start of the simulations. The spread of the results (error bars in the picture) tend to increase with the strength of the instability. For large $\sigma_{E 0}$ the instability is suppressed and the energy spread at the exit tends to $C \sigma_{E 0}$ where $C$ is the compression factor.

An interesting finding of our study concerns the impact on microbunching of the spreader distributing the beam to the two FELs at the end of the linac. The spreader under consideration for FERMI consists of two pairs of magnets, each pair delivering about $100 \mathrm{mrad}$ bending. For this study we considered two spreader designs. In the first ("unoptimized') design the two dipole pairs, arranged as perfect achromats, contribute $\Delta R_{56} \simeq 0.9 \mathrm{~mm}$ through the spreader. On the surface such a small value for $\Delta R_{56}$ would seem innocuously small but a closer look reveals that the resulting gain curve for the microbunching instability can be surprisingly high. The gain curve for the linac section from the bunch compressor through the spreader for the One-BC lattice is shown in Fig. 2, as determined from linear theory (solid line) 212] and from the numerical solution of the Vlasov equation (dots) incidentally, the close agreement between the two calculations provides validation of our solver, at least in the regime where linear theory applies. The picture shows a large gain in the sub $\mu \mathrm{m}$ region peaked at about $\lambda \simeq 0.3 \mu \mathrm{m}$. The gain is almost completely due to space-charge (as we verified by turning off CSR) and can be explained in terms of
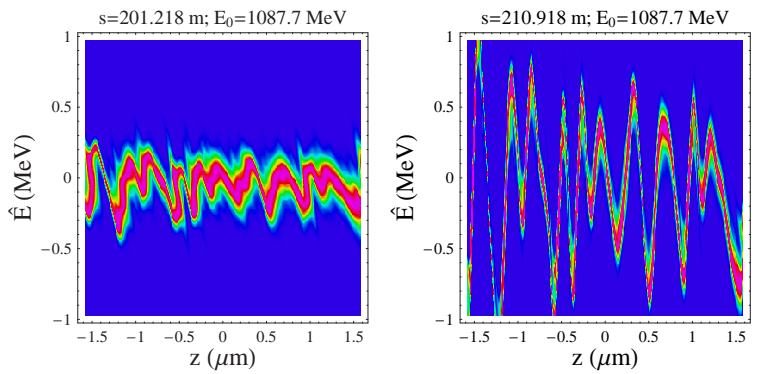

Fig. 4. Phase-space snapshots (uncorrelated energy vs. longitudinal position) at the end of the spreader (left figure) and end of the linac for the FERMI One-BC lattice (un-optimized spreader design); $\sigma_{E 0}=10 \mathrm{keV}, I_{f}=0.8 \mathrm{kA}$.

the properties of the longitudinal impedance model (1). At higher energies the location in the frequency spectrum of the space-charge impedance peak moves toward smaller wavelengths, with the peak value occurring at about $k r_{b} / \gamma \simeq 1$. Fig. 3 shows a comparison between the spectra of the space-charge impedance for the beam in the bunch compressor (red curve) and toward the exit of the linac (black curve). The transverse beam size assumed for the black curve is for the beam in the spreader where the beam is intentionally squeezed to minimize effects on the transverse emittance. At beam energy $E \sim 1 \mathrm{GeV}$ (end of the linac) $\hat{Z}$ peaks in the $\lambda \lesssim$ $1 \mu \mathrm{m}$ spectrum region when $r_{b} \lesssim 300 \mu \mathrm{m}$. The peak value $\hat{Z}_{\text {peak }} \sim 0.4 \times Z_{0} / \pi \gamma r_{b}$ scales inversely with $\gamma$ but also with the transverse size $r_{b}$. At the end of the FERMI linac $\hat{Z}_{\text {peak }}$ is still significant as the large beam energy is offset by small transverse beam sizes. A small relative rms energy spread (because of the large energy at the end of the linac) provides limited phase-space mixing causing negligible attenuation of the gain function (and also causing the wavelength $\lambda \simeq 0.3 \mu \mathrm{m}$ of the gain function peak to overlap with that of the space-charge impedance). The effect of the spreader on the beam phase-space is to cause an energy modulation at the $\mathrm{MeV}$ level (see Fig. 4).

An effective way to reduce the instability is to modify the spreader design to minimize $\Delta R_{56}$. This can be achieved in two ways: by reducing the dipole length (while increasing the magnetic field in order to maintain the same bending angle) and locally compensating $\Delta R_{56}$ with a suitable setting of the dispersion function within the dipoles (which has the effect of spoiling the achromatic properties of each dipole pair). Notice that reducing the dipole length (and hence the radius of curvature $\rho$ ) does 

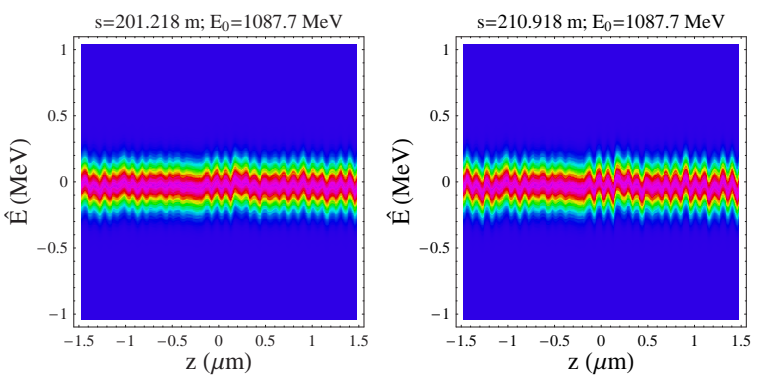

Fig. 5. As Fig. 4 but with optimized spreader design.

not worsen the CSR effects as for a fixed bend angle $\Delta \theta$ these scale as $\rho^{1 / 3} \Delta \theta$. For this study we have used both strategies and considered a second ('optimized') spreader design yielding $\Delta R_{56}<5 \mu \mathrm{m}$ from end to end of the spreader and a maximum local deviation within the spreader smaller than $40 \mu \mathrm{m}$. The resulting gain curve for microbunching (not shown here) has a peak value of only a few units and causes no noticeable impact on the beam, as shown in Fig. 5. 1

Although a careful investigation of the dependence of our results on the numerics (and in particular the choice of the grid size for representing the beam density) gives us some confidence that these results represent believable solutions of the Vlasov equation, there remains a question on whether the model being used, specifically that of longitudinal space charge, is sufficiently accurate. There are several approximations involved: the model is based on the assumption of an axis-symmetric beam with uniform transverse density and yields the on-axis longitudinal electric field in response to a purely longitudinal perturbation. An underlying assumption is that one can ignore fluctuations in the transverse density. This $1 \mathrm{D}$ model is a good approximation in regions of the spectrum corresponding to a length scale $\lambda / 2 \pi$ large compared to $r_{b} / \gamma$. However, the $1 \mathrm{D}$ model breaks down when $2 \pi r_{b} / \lambda \gamma$ becomes comparable to or is larger than $\sim 0.5[10$. This includes the region of the spectrum where the impedance (1) reaches its peak. Through the bunch compressors in the FERMI lattice we are generally in a regime where the relevant frequency band of the space charge impedance is safely on the side where the 1D approximation holds (as the peak of the impedance falls in a region of the frequency spectrum where microbunching is effectively suppressed

\footnotetext{
1 This was the spreader design used for the calculations of Fig. [1]
}

by the beam energy spread). This is not the case at the end of the linac where the relative energy spread is small and a modest $\Delta R_{56}$ through the spreader suffices to amplify frequencies in the very high frequency end of the spectrum.

While it is comforting to know that if the longitudinal space-charge electric field in the sub $\mu \mathrm{m}$ region is indeed as intense as predicted by the $1 \mathrm{D}$ model there exist technical solutions to minimize the microbunching in the spreader (by controlling $\left.\Delta R_{56}\right)$ more investigations are required to determine if these are actually needed. A numerical comparison between the field as predicted by the 1D model and evaluated from distributions of macroparticles using a full 3D Poisson solver could help settle this point and is in our plans. (Macroparticle simulations for the FERMI lattices carried out so far [5] are inconclusive as the grid resolution for solving the Poisson equation in those simulations was insufficient).

In conclusion, we have shown that direct techniques to solve the Vlasov equation represent a viable method for the study of the microbunching instability evolving from shot noise. While more work is needed to assess the reliability of the $1 \mathrm{D}$ model of space charge used in the solver the evidence so far is that the One-BC lattice proposed for FERMI should meet the specified $\sigma_{E}<150 \mathrm{keV}$ beam-quality requirement.

Useful discussions with M. Cornacchia, S. Di Mitri, W. Fawley, J. Qiang, and R. Warnock are gratefully acknowledged. Work supported by Department of Energy Contract No. DE-AC02$05 \mathrm{CH} 11231$.

\section{References}

[1] E.L. Saldin, et al., NIM-A, 483, 516 (2002).

[2] S. Heifets, G. Stupakov, and S. Krinsky, Phys. Rev. ST Accel. Beams 5, 064401 (2002).

[3] Z. Huang, et al., Phys. Rev. ST Accel. Beams 7, 074401 (2004).

[4] M. Venturini, Phys. Rev. ST Accel. Beams 10, 104401 (2007).

[5] J. Qiang, et al., PAC07, Proceedings (2007).

[6] M. Venturini, R. Warnock, and A. Zholents, PRST-AB 10, 054403 (2007).

[7] M. Venturini and R. Warnock, PRL 89, 224802 (2002); M. Venturini, et al., Phys. Rev. ST Accel. Beams 8, 014202 (2005).

[8] G. Dattoli, et al., NIM-A, 574, 244 (2007); M. Migliorati and A. Schiavi, private communication.

[9] J. Rosenzweig, et al., NIM-A., 393, 376 (1997). 
[10] M. Venturini, Phys. Rev. ST Accel. Beams 11, 034401 (2008)

[11] C. Bocchetta, et al., FERMI@Elettra: a Seeded Harmonic Cascade FEL for EUV and Soft X-Rays, Proc. of the FEL Conf. 2006, Berlin, Germany. To appear.

[12] Z. Huang and K.-J. Kim, PRST-AB 5, 074401 (2002).

This document was prepared as an account of work sponsored by the United States Government. While this document is believed to contain correct information, neither the United States Government nor any agency thereof, nor The Regents of the University of California, nor any of their employees, makes any warranty, express or implied, or assumes any legal responsibility for the accuracy, completeness, or usefulness of any information, apparatus, product, or process disclosed, or represents that its use would not infringe privately owned rights. Reference herein to any specific commercial product, process, or service by its trade name, trademark, manufacturer, or otherwise, does not necessarily constitute or imply its endorsement, recommendation, or favoring by the United States Government or any agency thereof, or The Regents of the University of California. The views and opinions of authors expressed herein do not necessarily state or reflect those of the United States Government or any agency thereof, or The Regents of the University of California.

Ernest Orlando Lawrence Berkeley National Laboratory is an equal opportunity employer. 